\title{
A framework for Cloud Computing Adoption by Saudi Government overseas agencies
}

\author{
Ahmed Albugmi \\ School of Electronics and Computer Science \\ University of Southampton \\ Southampton, United Kingdom \\ ana1a14@soton.ac.uk
}

\author{
Robert Walters, Gary Wills \\ School of Electronics and Computer Science \\ University of Southampton \\ Southampton, United Kingdom \\ (rjw1-gbw)@ecs.soton.ac.uk
}

\begin{abstract}
This study aims to identify key factors that organizations should consider when deciding whether to adopt cloud computing, and sets out a framework for how these factors can be weighed in order to make a decision. The study uses the Saudi Government agencies as a case study and makes several specific recommendations that pertain to the Saudi authorities' implementation of cloud computing.

Although there are many benefits associated with deployment of cloud computing applications, there are also several challenges, such as compliance, legal issues, hosting issues, security, trust and privacy. There are also inadequate resources and guidelines for the policy makers and managers to inform their decision of whether or not to adopt cloud computing.

This study identifies a number of factors; technological, environmental, organizational and societal, which need to be considered when an organization decides whether or not to adopt cloud computing. After identifying these factors, the study develops a comprehensive framework for organizations to assess their readiness for cloud computing. In addition, the feasibility of cloud computing applications is assessed so that different delivery and deployment models can be taken into account, and cloud computing evaluated from both business and customer perspectives.
\end{abstract}

Keywords- Cloud computing, Adoption,technologyorganisation-environment

\section{INTRODUCTION}

There are many issues that can contribute to the adoption or rejection of cloud computing, including compliance and legal challenges, security challenges and other organizational based aspects. Which issues are most salient, and which decision is ultimately made, depends greatly on the specific socio-cultural context.

The adoption of cloud computing thus depends on how a given organization measures the risks in a given case, as well as the available forms of mitigation and the nature of the risk itself. If customers can identify the risks in a given situation and make informed decisions as to what degree of risk they are prepared to accept, then they will feel safer and more secure when using cloud computing technology. The prospect of contributing to better understanding and decision-making in this way is the reason that this work appeals to the researcher, who aims to build a framework of factors which influence the adoption of cloud computing.

The risks associated with the adoption of cloud computing can be illustrated by considering historic examples. However, several instances of possible power outages of cloud and even the loss of service are a cause for concern which has created a feeling of distrust with the technology.
It is important to consider risks that arise in connection with the installation of cloud computing. Installation can be facilitated via different means and various challenges can be addressed. These different methods of installation approaches should be appropriate to cover general aspects of computing. Taken together, the process of installation designed to implement cloud computing must be one which covers the evaluation of all challenges. It should also highlight any critical points which may potentially pose problems and complications in the delivery of services to customers through the deployment of cloud computing. In addition, it must provide solutions which minimize the occurrence of these problems on the adoption of cloud computing.

\section{LITERATURE REVIEW}

\section{A) Overview of Cloud Computing Adoption}

The advent of cloud computing technologies constitutes a significant milestone in the history of information systems. The National Institute of Standards and Technology (NIST) defines cloud computing as follows:

"Cloud computing is a model for enabling convenient, on demand network access to a shared pool of configurable computing resources that can be rapidly provisioned and released with minimal management effort or service provider interaction" [1].

Cloud computing has received attention from the stakeholders of business organizations, institutions, information technology companies and academicians. Moreover, the major investment organizations are putting their trust and investment in cloud computing and this is mainly due to cloud computing being quoted as "A new economic model of cloud computing for communication and information technology (CIT)." In this model, the computing is allowed to bypass an investment i.e., there is no need to invest in limited internally managed IT resources. However, the working company needs "to outsource and chase the needs of IT resources and back up by using cloud computing as a provider and pay as they use." This new archetype of computing has posed many challenges to the organizations who are considering adopting it. The major identified challenges include; security, trust, compliance, legal and other organizational management challenges. Almost all of the challenges given here are in relation to trust. 
Cloud computing, along with various delivery models and deployment clouds, offers remuneration benefits to the business community in general and businesses as a whole [2]. Moreover, the major benefits associated with cloud computing include low maintenance costs, low administrative costs and economies of scale arising from the reduced cost of the IT infrastructure. Other benefits include improved performance through scalable computing, and unlimited storage and memory capabilities. Further, the efficiency of the computing system, low cost to undertake security measures, quick incident response and easier data monitoring are also important factors.

\section{B) Organizational context}

Organizational factors affecting the technological adoption include the behavior of leadership, the decision making power of management, leadership controls and links of the organization within and outside of the organization. The modes of communication prevalent and the dependencies of the organization on these modes also play a fundamental role in increasing the adoption of the technological applications and innovations [3]. The new technologies help the organization to forge better communication channels within the organization and strengthen the inter-organizational linkages; therefore, the organizations are more dependent on the innovative methods and applications to enhance the communication [4]. For the effective introduction of the new technologies it is paramount that the leadership of the organization understands the importance of the technological innovation and is willing to implement it [4]. For that purpose, the human resource management department of the organization and top executives must collaborate in their efforts to change the attitudes of employees and consumers and prepare them for the new technologies being introduced, in order to increase the performance and productivity of both the staff and the organization as a whole. Consequently, the managers of the organization require change management expertise, otherwise the implementation of the technologies cannot be made [5].

\section{C) Environmental context}

The environment of the organization represents the stakeholders, competitors, consumers, governmental agencies, community and suppliers etc. These factors determine the needs for new technologies and innovations for the organization and identify the available resources which can be potentially used to introduce and implement the new technologies [6]. The foregoing factors can either encourage or discourage the adoption of the technologies necessary for the innovation and development of the organization. The ever evolving consumers' needs, the market structure and competitive landscape prod the organizations into using the various technological innovations [7]. Government regulation is another important factor which can either block or help propagate the technological innovations. The physical location (hosting the data management applications such as cloud computing) of new technologies is another important aspect which determines whether the consumers of the organization would support the implementation of new technologies [8].

\section{D) Technological context}

Tornatzky and Fleischer described the influence of technology within the 'systems design perspective' which involves the system's life cycle, technical expertise, socio-centric and techno-centric approaches of the organization [9]. The techno-centric approach requires the organization to understand the importance of the technologies in overhauling their systems and the innovations of their processes and products [10]. The experience of the organization in importing and implementing the new technologies matters a great deal in this context, along with the expertise of effectively engineering and installing the new systems. (Ibid). However, the socio-centric approach is related to the construction and redesign of the organizational systems, modifying the HR policies to accommodate the new technological ideas, redesigning the jobs and successfully installing and integrating the technological innovations. The presence of technological sustainability and its continuous use is also important for the continuous use of the newly integrated technologies within the organization [11].

As this framework considers the influence of the organizational, technological and environmental factors, it has the flexibility to fulfill the criterion of inclusion of all possible factors to determine the feasibility of technological adoption at the organizational level [12]. Despite the advantages of the TOE framework for the adoption of the technological innovations for the organizations, the applications of this model should be considered carefully while applying differing social perspectives in different countries. In almost every society there are norms and values which can affect the organizational structure, management styles, modes of change management mechanisms, the technological expertise and experience of using the new technologies. Therefore, this framework may involve differing factors in different countries based upon their socio-centric, techno-centric and conflict management approaches [13].

\section{THE PROPOSED FRAMEWORK}

The proposed framework is intended to investigate the most important factors that influence the adoption of cloud computing in Saudi agencies oversees. This framework formulates the categories of new IT based technologies involving the factors which can assist or hamper the use of information systems within organizations, based on their environmental or technological contexts.

The framework factors were identified by critically reviewing studies found in the literature together with factors from the IT standards within the context of Saudi Government overseas agencies. These are illustrated in the cloud computing adoption framework. (Fig1). 


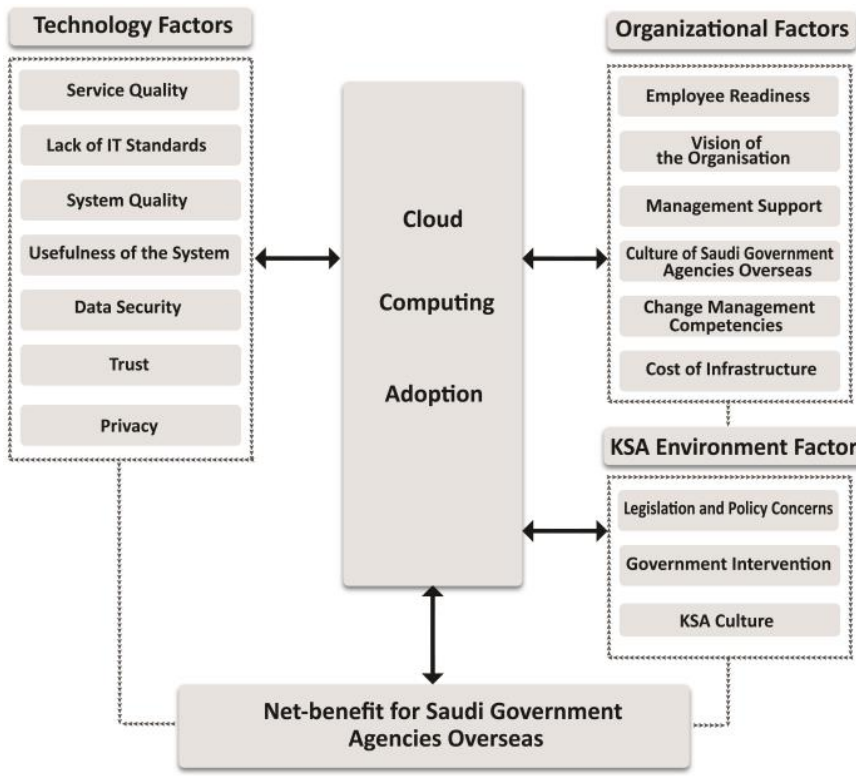

Figure 1: The proposed framework for adoption of cloud computing in Saudi Government agencies overseas

The hypotheses formulated for this study are given below:

\section{A. Organizational Factors}

- Employee readiness The organization promotes the usage of cloud computing through training and enhancing the experience of the employees with the use of cloud computing. Furthermore, if the employees are not fully trained and prepared to manage cloud computing services and deliver them to the end-users, the intention to adopt cloud computing can be aborted. Kuo found that the decision to adopt cloud computing for the improvement of health care services was affected by the level of expertise possessed by the staff [14]. The management of the organization may face failure in successfully implementing cloud computing if the factor of employee readiness has not been taken into consideration prior to the adoption of cloud computing.

- Management support Management support is another key factor which has the potential to affect the decision of adopting cloud computing. The visionary and ambitious management team of an organization can perceive the benefits of the new technologies and their implementation in the organization. Marston rated management support as a key factor which can affect the decision of adoption of cloud computing [15].

- Culture of the organization The organizational culture can determine the success or failure of the adoption of cloud computing. Twati and Gammack showed that organizational culture in Libya discouraged the use and adoption of cloud computing
[16]. This was because the employees of many organizations were not familiar with data management via cloud computing.

- The vision of the organization Previous research in the field of information systems argued that early adoption of a technological innovation is based on an organization's vision. This vision serves key functions in interpretation, legitimization and the organization and mobilization of economic roles and exchanges to adopting new technology. Ngai found that the vision of the organization and management support were the most frequently cited critical factors to the successful implementation of new systems [17].

When an organization intends to change the technologies to innovate its processes and products, the change management strategies hold critical value in respect of changing the behavior of the employees and consumers towards the newly installed technological applications.

- Cost of infrastructure The infrastructure costs can affect the decision to adopt cloud computing. Moreover, organizations with limited resources would not be able to adopt and subsequently use cloud computing. Marston found that if the cost of infrastructure exceeds the available financial resources of the organization, the decision to adopt cloud computing cannot made by the organization's managers [18].

\section{B. Technology Factors}

- User's trust User's trust is paramount when making the decision of whether or not to adopt cloud computing. Cloud computing applications offer a landscape of opportunities and challenges for the organization and users who adopt it. For most of the organizations, the adoption of cloud computing is a major concern, which not only arises from the providers' of cloud computing applications, but also from the capabilities that cloud computing has to offer [19].

- Service quality The quality of the services available through cloud computing applications is another important factor affecting the decision of whether to adopt cloud computing applications. Several studies reported the positive influence of service quality on the decision of whether to adopt cloud computing services in the organization [20].

- System quality System quality is increased by scaling cloud computing applications to meet the organizational and user requirements. High system quality also increases the user's satisfaction with the 
services provided by cloud computing applications. Armbrust showed that high system quality can increase the usage of cloud computing among employees of the organizations, showing system quality as key factor for the adoption of cloud computing [21].

- Usefulness of the system Usefulness of the system is another key factor which has the potential to affect the decision of adopting cloud computing. Furthermore, cloud computing will be adopted only when the consumers consider it as useful for processing the required functions of current systems.

- User's data security Data security is a major issue faced by the management of organizations which impacts the decision of whether to adopt cloud computing. For the adoption of e-Health cloud computing, AbuKhousa found that data security positively affected the adoption of cloud computing [22].

- User Privacy User privacy is as an important concern for the leadership of an organization when making the decision of whether or not to adopt cloud computing. Alsanea found impact that cloud clouding applications had on privacy within organizations [23].

- Lack of IT standards While other industries, such as the computer and financial industries, have long established IT standards for adopting new technology, government sectors have lagged behind. The lack of standards makes it difficult for an organization to adopt cloud computing. The lack of IT standards may have a negative impact on the adoption of innovative technology.

\section{Environmental Factors}

- Legislation and policies concerns Government policies and legislation regarding how cloud computing applications are imported and implemented can affect the decision of whether or not to adopt cloud computing within an organization. Legislation and policies may support or forbid cloud computing due to challenges in implementation, including data security and privacy. Several studies have observed the impact that legislation and policy concerns have on the decision of whether or not to adopt cloud computing. Bernsmed reported that legislation and policy concerns were found to be one obstacle which affects the adoption of cloud computing in health services. [24].

- Government intervention Government intervention is another challenge facing public sector organizations when considering whether to adopt cloud computing applications. The government will either support or reject the adoption of cloud computing in public organizations.

- KSA Culture Existing cultural conditions always determine whether, when, how and in what form a new innovation will be adopted. Moreover, argues that many of the differences in management styles and organizational practices of companies throughout the world can be related to differences in the collective mental programming of people in different national cultures. Alhammadi revealed that the cultural dimensions of Saudi Arabia influence the adoption of IT [25].

\section{CONCLUSION}

This paper sets out the scope and research aims for a new study of the various factors affecting the adoption of cloud computing, using the potential adoption of cloud computing by Saudi Government agencies overseas as a case study. It introduces the topic and sets out key research aims and questions, discusses the relevant literature on the adoption of cloud computing, presents a theoretical framework within which to address its aims and explains research methods through which the aims will be achieved.

Future work will address the issues and challenges associated with cloud computing applications by application of a theoretical framework and through analysis of the results of the survey to a thorough analysis of the available literature. This study will build on existing work in the area of cloud computing adoption for overseas agencies, with a view to promoting the adoption of cloud computing worldwide. In particular, it will contribute to information systems research by providing a theoretical framework for the use and acceptance of cloud computing.

It is therefore essential to understand these challenges from the perspective of government organizations, in order to highlight the areas important to enacting the necessary changes.

The findings of this study will have theoretical and practical implications for developing nations and especially Saudi Arabia. It will identify and analyze the relationship between Saudi Arabia's technological, organizational and environmental contexts and the acceptance of cloud computing by Saudi Government agencies. The findings of this research will provide a practical guideline for the successful adoption of cloud computing in Saudi Government agencies overseas.

By applying a new framework which takes account of factors that are especially salient in the context of Saudi Arabia, the study will assist other developing countries which are planning to implement cloud computing in similar circumstances. In particular, it will help other developing countries to determine relevant factors for consideration prior to adopting cloud computing. 
A final benefit of the study, which is perhaps easy to neglect, will accrue to the participants. The study will offer respondents an opportunity to reflect on their expectations about cloud computing in Saudi Arabia.

\section{REFERENCES}

[1]. NIST (2016) NIST Cloud Computing Program. National Institute of Standards and Technology, United States. Available from: http://www.nist.gov/itl/cloud/ [Accessed 15th March 2016]

[2]. Gong, C., Liu, J., Zhang, Q., Chen, H. \& Gong, Z. 2010, "The Characteristics of Cloud Computing", pp. 275.

[3]. Del Aguila-Obra, A. R., \& Padilla-Melendez, A. (2006). Organizational factors affecting Internet technology adoption. Internet research, 16(1), 94-110.

[4]. Thamhain, H. J. (2005). Management of technology: Managing effectively in technology-intensive organizations. US: John Wiley \& Sons Inc.

[5]. Ming-Ju Pan, \& Woan-Yuh Jang. (2008). Determinants of the adoption of enterprise resource planning within the technology-organization-environment framework: Taiwan's communications industry. The Journal of Computer Information Systems, 48(3), 94.

[6]. Low, C., Chen, Y., \& Wu, M. (2011). Understanding the determinants of cloud computing adoption. Industrial management \& data systems, 111(7), 1006-1023.

[7]. Yee-Loong Chong, A., \& Ooi, K. B. (2008). Adoption of interorganizational system standards in supply chains: an empirical analysis of RosettaNet standards. Industrial Management \& Data Systems, 108(4), 529. 547.

[8]. Xu, S., Zhu, K., \& Gibbs, J. (2004). Global technology, local adoption: A Cross-Country investigation of Internet adoption by companies in the United States and china. Electronic Markets, 14(1), 13-24.

[9]. Tornatzky, L. G., Fleischer, M., \& Chakrabarti, A. K. (1990). Processes of technological innovation. Lexington Books.

[10]. Hameed, M. A., Counsell, S., \& Swift, S. (2012). A conceptual model for the process of IT innovation adoption in organizations. Journal of Engineering and Technology Management, 29(3), 358-390.

[11] . Baker, J. (2012). The technology-organizationenvironment framework. InInformation systems theory (pp. 231-245). Springer New York.

[12] . Oliveira, T., \& Martins, M. F. (2011). Literature review of information technology adoption models at firm level. The Electronic Journal Information Systems Evaluation, 14(1), 110-121.

[13] .Xu, S., Zhu, K., \& Gibbs, J. (2004). Global technology, local adoption: A Cross-Country investigation of Internet adoption by companies in the United States and china. Electronic Markets, 14(1), 13-24.

[14]. Kuo, A.M. 2011, "Opportunities and challenges of cloud computing to improve health care services", Journal of medical Internet research, vol. 13, no. 3, pp. e67.

[15]. Marston, S., Li, Z., Bandyopadhyay, S. \& Ghalsasi, A.
2011, "Cloud Computing - The Business Perspective", , pp. 1.

[16].Twati, J. M., \& Gammack, J. G. (2006). The impact of organisational culture innovation on the adoption of IS/IT: the case of Libya. Journal of enterprise information management, 19(2), 175-191.

[17]. Lin, C., Ramakrishnan, K.K., Liu, J. \& Ngai, E. 2016, "Guest Editorial Special Issue on Cloud Computing for IoT", IEEE Internet of Things Journal.

[18]. Marston, S., Li, Z., Bandyopadhyay, S. \& Ghalsasi, A. 2011, "Cloud Computing - The Business Perspective", , pp. 1.

[19]. Alsanea, M., Jennifer Barth \& R. Griffith. (2014, December). Factors Affecting the Adoption of Cloud Computing in the Government Sector: A Case Study of Saudi Arabia, 2014 IEEE 6th International Conference on (pp. 1040-1044). IEEE.

[20]. Ardagna, D., Casale, G., Ciavotta, M., Pérez, J.F. \& Wang, W. 2014, "Quality-of-service in cloud computing: modeling techniques and their applications", Journal of Internet Services and Applications, vol. 5, no. 1, pp. 1-17.

[21] .Armbrust, M., Fox, A., Griffith, R., Joseph, A. D., Katz, R., Konwinski, A., \& Zaharia, M. (2010). A view of cloud computing. Communications of the ACM, 53(4), 50-58.

[22] .AbuKhousa, E., Mohamed, N., \& Al-Jaroodi, J. (2012). e-Health cloud: opportunities and challenges. Future Internet, 4(3), 621-645.

[23] .Alsanea, M., Jennifer Barth \& R. Griffith. (2014, December). Factors Affecting the Adoption of Cloud Computing in the Government Sector: A Case Study of Saudi Arabia, 2014 IEEE 6th International Conference on (pp. 1040-1044). IEEE.

[24].Bernsmed, K., Cruzes, D. S., Jaatun, M. G., Haugset, B., \& Gjære, E. A. (2014, September). Healthcare Services in the Cloud--Obstacles to Adoption, and a Way Forward. In Availability, Reliability and Security (ARES), 2014 Ninth International Conference on (pp. 158-165). IEEE.

[25].Abdullah Alhammadi, Clare Stanier \& Alan Eardley 2015, "THE DETERMINANTS OF CLOUD COMPUTING ADOPTION IN SAUDI ARABIA", Computer Science \& Information Technology, vol. 5, no. 14 , pp. 55-67. 